\title{
Renal artery stenosis: epidemiology and treatment
}

This article was published in the following Dove Press journal: International Journal of Nephrology and Renovascular Disease 13 May 2014

Number of times this article has been viewed

\author{
Benjamin R Weber \\ Robert S Dieter \\ Loyola University Medical Center, \\ Maywood, IL, USA
}

Correspondence: Robert S Dieter Loyola University Medical Center, 2160 S Ist Ave, Maywood, IL 60I53, USA Email rdieter@lumc.edu
Abstract: Renal artery stenosis (RAS) is a frequently encountered problem in clinical practice. The disease encompasses a broad spectrum of pathophysiologies and is associated with three major clinical syndromes: ischemic nephropathy, hypertension, and destabilizing cardiac syndromes. The two most common etiologies are fibromuscular dysplasia and atherosclerotic renal artery disease with atherosclerotic disease accounting for the vast majority of cases. Atherosclerotic renovascular disease has considerable overlap with atherosclerotic disease elsewhere and is associated with a poor prognosis. A wide range of diagnostic modalities and treatment approaches for RAS are available to clinicians, and with the advent of endovascular interventions, selecting the best course for a given patient has only grown more challenging. Several clinical trials have demonstrated some benefit with revascularization but not to the extent that many had hoped for or expected. Furthermore, much of the existing data is only marginally useful given significant flaws in study design and inherent bias. There remains a need for further identification of subgroups and appropriate indications in hopes of maximizing outcomes and avoiding unnecessary procedures in patients who would not benefit from treatment. In recent decades, the study of RAS has expanded and evolved rapidly. In this review, we will attempt to summarize the amassed body of literature with a focus on the epidemiology of RAS including prevalence, overlap with other atherosclerotic disease, and prognosis. We will also outline existing diagnostic and treatment approaches available to clinicians as well as summarize the findings of several major clinical trials. Finally, we will offer our perspective on future directions in the field.

Keywords: atherosclerosis, hypertension, nephropathy, renovascular, stent, angioplasty, endovascular

\section{Introduction}

Renal artery stenosis (RAS) is general term that refers to any vascular lesion causing narrowing of the renal artery thereby impairing blood flow to the kidney. This disease encompasses a broad range of pathophysiologies, the two most common being fibromuscular dysplasia (FMD) and atherosclerotic renal artery disease. ${ }^{1}$ This review will primarily focus on the epidemiology and treatment of atherosclerotic renal artery stenosis (ARAS), which accounts for the vast majority of cases. RAS is associated with three major clinical syndromes: ischemic nephropathy, hypertension, and destabilizing cardiac syndromes. However, a diagnosis of RAS may also result from an incidental finding in an otherwise asymptomatic patient. A diagnosis of ARAS is associated with a poor prognosis and often with atherosclerotic disease in 
other vascular beds. A wide range of diagnostic modalities and treatment approaches for RAS are available to clinicians, and with the advent of endovascular interventions, selecting the best course for a given patient has only grown more challenging. Several clinical trials have demonstrated some benefit with revascularization but not to the extent that many had hoped for or expected. ${ }^{2,3}$ Furthermore, much of the existing data is only marginally useful given significant flaws in study design and inherent bias. There remains a need for further identification of subgroups and appropriate indications in hopes of maximizing outcomes and avoiding unnecessary procedures in patients who would not benefit from treatment. In recent decades, the study of RAS has expanded and evolved rapidly. In this review, we will attempt to summarize the amassed body of literature with a focus on the epidemiology of RAS including prevalence, overlap with other atherosclerotic disease, and prognosis. We will also outline existing diagnostic and treatment approaches available to clinicians as well as summarize the findings of several major clinical trials. Finally, we will offer our perspective on future directions in the field.

\section{Nonatherosclerotic renal artery disease}

Nonatherosclerotic renal artery disease accounts for a wide range of etiologies. This list includes FMD, aneurysms, congenital or traumatic arteriovenous fistulas, vasculitis, neurofibromatosis, trauma, embolization, congenital bands, post-radiative therapy, and dissection. FMD is by far the most common of the nonatherosclerotic causes, accounting for $10 \%$ of all RAS. ${ }^{1}$ FMD predominately affects premenopausal woman, typically ranging from 15 to 50 years of age and has been associated with a history of smoking and hypertension. ${ }^{4}$ Unlike atherosclerotic renovascular disease, FMD is largely a disease of the young and healthy with few cardiovascular risk factors. Histologically, FMD may involve the intima, media, and adventitia; however, 90\% involve the media. ${ }^{1}$ On angiography, FMD has classically been described as having a "beads-on-a-string" appearance due to contrast filling of consecutive aneurysms along the renal artery. In patients with FMD, the distal two-thirds of the renal artery is the most commonly affected location; however, the disease may also involve carotid and vertebral arteries. ${ }^{5}$ Though it may resemble vasculitis, FMD is noninflammatory, and the cause remains unknown. It is thought that a genetic component plays a role. ${ }^{4} \mathrm{FMD}$ is generally associated with a good prognosis and usually does not progress to complete occlusion.

\section{Atherosclerotic renal artery disease}

Atherosclerotic disease is the most common disease to affect the renal arteries, making up $90 \%$ of all renovascular lesions. ${ }^{1}$ Atherosclerotic RAS (ARAS) typically involves the proximal third of the renal artery including the perirenal aorta and ostium. ${ }^{5}$ ARAS is associated with renovascular hypertension, but more consistently, ARAS presents with nephropathy. ${ }^{6}$ Unlike FMD, patients with ARAS are commonly elderly and have multiple cardiovascular risk factors. In these patients, atherosclerosis is generally systemic and not limited to the renal artery, a concept to be discussed in depth in a following section. More often than not, ARAS is a progressive disease characterized by worsening stenosis and eventual occlusion with wider implications in regards to both organ function and patient prognosis.

\section{Pathophysiology}

RAS is a common cause of secondary hypertension. Though likely an oversimplification of a more complex pathophysiology, the mechanism leading to the development of renovascular hypertension is typically classified as either renin-dependent or primarily a result of volume overload. This theory dates back to the 1930s, when Goldblatt et al performed a series of studies examining the impact of unilateral and bilateral RAS (BRAS) on blood pressure. ${ }^{7}$ By clamping renal arteries in dogs, Goldblatt et al demonstrated a systemic pressor effect. They postulated that this effect was due to a substance produced by the kidneys causing vasoconstriction. The substance was ultimately isolated and identified as the proteolytic enzyme now known as renin. Renin is an early effector in the larger renin angiotensin aldosterone neurohormonal cascade. When ischemia occurs downstream of a stenotic renal artery, renin is released from juxtaglomerular cells. Renin then cleaves angiotensinogen to form angiotensin I, which must be further processed to angiotensin II by angiotensin-converting enzyme (ACE) produced in the lung endothelium and vasculature. Angiotensin II is the active enzyme and has multiple downstream effects. Angiotensin-II-mediated vasoconstriction causes hypertension, leading to pressure diuresis of the unaffected kidney. Glomerular filtration rate (GFR) is increased via vasoconstriction of the efferent arteriole. Antidiuretic hormone is released from the posterior pituitary gland, causing water conservation, further contributing to pressure diuresis. Release of aldosterone from the adrenal glands enhances exchange of sodium in the nephron-promoting volume retention. In addition, angiotensin increases sympathetic tone. The mechanism 
behind BRAS or unilateral RAS with a solitary kidney is due to extracellular fluid overload secondary to decreased diuresis rather than a renin-mediated mechanism. It is important to distinguish between renin-dependent and renin-independent patients as the initiation of an angiotensin II inhibitor may induce acute renal failure in a renin-dependent system.

Ischemic nephropathy can be defined as an obstruction causing decreased perfusion leading to renal ischemia and subsequent excretory dysfunction. The cause of ischemic nephropathy has not been fully elucidated. However, several interrelated mechanisms have been proposed explaining how a hemodynamically significant lesion ultimately results in interstitial fibrosis. ${ }^{8}$ By one pathway, recurrent local ischemia causes tubulointerstitial injury and microvascular damage. By another, global hypoperfusion of the kidney leads to altered endothelial and epithelial factors as well as activation of the renin-angiotensin aldosterone system and subsequent vasoconstriction. Both pathways are thought to contribute to oxidative injury, increased production of fibrogenetic cytokines, and inflammation, ultimately leading to atrophy and fibrosis.

RAS may either cause or exacerbate cardiac destabilizing syndromes, including unstable angina (UA) and congestive heart failure characterized by flash pulmonary edema. RAS precipitates these conditions through three general mechanisms: volume overload, peripheral arterial vasoconstriction, and direct effects of angiotensin on the myocardium. Flash pulmonary edema can be described as a specific presentation of acute decompensated heart failure characterized by rapid fluid accumulation within the lungs. Flash pulmonary edema can occur secondary to several conditions, all of which result from an acute increase in end diastolic left ventricular pressure. Flash pulmonary edema does occur in unilateral RAS, but tends to occur more often in patients with BRAS. ${ }^{9}$ In BRAS, the mechanism can be explained by impaired natriuresis and thus, a propensity for volume overload. This phenomenon was first reported by Pickering et al in 1988 in a case series of eleven hypertensive patients with BRAS and recurrent pulmonary edema. ${ }^{10} \mathrm{UA}$ is defined as pain due to cardiac ischemia that is new in onset or is increasing in frequency or intensity. The prototypical cause of UA is atherosclerosis and plaque rupture. RAS, however, may contribute to UA via an acute angiotensin-mediated increase in afterload. Increased left ventricular workload leads to increased oxygen demand, resulting in myocardial ischemia.

\section{Prevalence}

Historically, it has been difficult to determine the true prevalence of RAS in the general population. Prior to newer, noninvasive diagnostic techniques, most studies were performed postmortem or were selective of subpopulations undergoing angiography. For example, several decades ago, a large series of 14,152 patients who underwent angiography found insignificant disease $(<50 \%$ stenosis $)$ in one or more vessels in $5.1 \%$ of patients and significant stenosis in $6.3 \%$ of patients. ${ }^{11}$ A more recent population-based study utilizing a noninvasive screening technique found similar results. The authors found that $6.8 \%$ of both Black and White elderly patients enrolled in the Cardiovascular Health Study had $\geq 60 \%$ stenosis or occlusion as determined by renal duplex sonography. ${ }^{12}$ There was no correlation between RAS and ethnicity while disease was independently associated with age, hyperlipidemia, and hypertension. It is well established that the prevalence of RAS is increased in elderly patients, particularly in those with additional comorbid conditions such as diabetes, aortoiliac occlusive disease, coronary artery disease (CAD), or hypertension. ${ }^{1,13}$

\section{Overlap of RAS with other atherosclerotic disease}

It has long been recognized that patients with known atherosclerotic vascular disease in one arterial bed are likely to have or develop disease in another. The risk of concurrent ARAS ranges from $26 \%$ to $50 \%$ with a diagnosis of arterial atherosclerotic disease elsewhere in the arterial vasculature. ${ }^{14}$ Specifically, the overlap of RAS with CAD, peripheral arterial disease (PAD), and carotid arterial disease has been well described. There also appears to be a relationship between the severity of RAS and the incidence of atherosclerotic disease. In a study by Wollenweber et al, $\sim 31 \%$ of patients with mild atherosclerotic narrowing ( $<50 \%$ occlusion) of a renal artery had symptomatic arterial disease in the coronary, cerebrovascular, or peripheral vascular circulation. ${ }^{15}$ This proportion was increased to $49 \%$ in patients with moderate to severe stenosis $(>50 \%)$.

In patients with CAD, the risk of RAS ( $>50 \%$ stenosis) ranges from $22 \%$ to $89 \%$. ${ }^{14}$ The prevalence of RAS tends to increase with the number of coronary vessels involved. In fact, the presence of significant CAD with greater than two vessel involvement was found to be an independent predictor of RAS with a sensitivity of 0.84 and specificity of $0.77 .{ }^{16}$ A history of percutaneous coronary intervention (PCI) is quite prevalent among patients with significant RAS, $\sim 39 \% .{ }^{17}$ Not surprisingly, clinically overt CAD is more common among patients with RAS than without. In a study of patients with unsuspected RAS, 58\% were shown to have clinical CAD (documented myocardial infarction, 
positive PCI, history of coronary artery bypass grafting, electrocardiogram change, or angina) compared with $39 \%$ of patients without RAS $(P=0.002) .{ }^{18}$

The overlap of PAD with RAS is significant. In a group of patients who underwent angiography as a part a routine evaluation for a known vascular pathology, greater than $50 \%$ RAS was found in $38 \%$ of patients with abdominal aortic aneurysm, 33\% with aorto-occlusive disease, and 39\% with lower-extremity occlusive disease. ${ }^{20}$ A prospective study of patients with RAS documented by ultrasonic duplex scanning demonstrated a similarly high prevalence of PAD as assessed by ankle/brachial systolic pressure ratio. ${ }^{21}$ For those with highgrade RAS, the prevalence of severe PAD was $73 \%$ versus $25 \%$ with RAS of less than $60 \%$. Another study observed a trend for patients with increasing degrees of renal artery disease to have increasing degrees of lower extremity arterial disease. ${ }^{22}$

Carotid artery lesions are more common and more severe in patients with renovascular hypertension. It has been shown that $40 \%$ to $46 \%$ of patients with significant RAS have moderate to severe occlusion of the carotid circulation. ${ }^{14} \mathrm{~A}$ case-control study of patients without history or symptoms of cerebrovascular disease (CVD) compared patients with renovascular hypertension to those with essential hypertension (EH) ${ }^{23}$ Almost twice as many patients with renovascular hypertension had carotid arterial stenosis as compared to those with EH. In the same study, it was found that the plaques in patients with renovascular hypertension were more heavily calcified than those with EH. Just as with PAD, increasing severity of RAS correlates with an increasing prevalence of CAD. From mild RAS to severe RAS, the prevalence increases from $7 \%$ to $28 \%$, a striking four-fold difference. ${ }^{22}$ There is also an association of RAS with clinical CVD. This relationship was examined in a series of autopsies of patients with clinical evidence of stroke, who died between 1980 and 1997. ${ }^{24}$ The authors identified significant atherosclerotic RAS ( $>75 \%$ ) in $10.4 \%$ of patients. Furthermore, patients with carotid artery stenosis were more than four times as likely to have RAS than patients without carotid artery stenosis ( $24.4 \%$ versus $5.9 \%, P=0.0001$ ). Conversely, $67.7 \%$ of patients with RAS had carotid artery stenosis. Others have found that a history of CVD is an independent predictor of the presence of RAS. ${ }^{17}$ In a large series of over 14,000 patients, a history of CVD was elicited in $9.2 \%$ without RAS versus $20.2 \%$ with RAS (odd's ratio: $2.3,95 \%$ confidence interval $[\mathrm{CI}]: 2.0-2.7, P=0.000001) .{ }^{17}$

\section{Prognosis}

ARAS is a progressive disease in regards to both lesion and kidney function, conferring a poor prognosis to affected patients. Caps et al followed the natural progression of RAS in 295 renal arteries in 170 patients with serial duplex scans. ${ }^{25}$ Stratified by initial degree of stenosis, the 3 -year cumulative incidence of renal artery disease progression was $18 \%$ in normal arteries, $29 \%$ of arteries with $<60 \%$ stenosis, and $49 \%$ of those with $\geq 60 \%$ stenosis at baseline. Total occlusion occurred in only nine $(6.3 \%)$ of the arteries with $\geq 60 \%$ stenosis. The authors concluded that while renal artery disease progression occurs frequently, progression to total renal artery occlusion does not. An alternative study observed progression to total occlusion in $39 \%$ of patients with $\geq 75 \%$ stenosis on renal arteriography. ${ }^{26}$ Unfortunately, complete occlusion occurs even in patients medically treated with adequate blood pressure control. In the Dutch Renal Artery Stenosis Intervention Cooperative (DRASTIC) trial, progression to complete occlusion occurred in $16 \%$ of patients treated medically. ${ }^{27}$ At baseline, several conditions have been shown to be significantly associated with renal artery disease progression, including severity of disease and comorbid conditions including diabetes and hypertension. Specifically, a stepwise Cox proportional hazards model included four baseline factors that were significantly associated with the risk of renal artery disease progression. ${ }^{25}$ These included systolic blood pressure $\geq 160 \mathrm{mmHg}$ (relative risk $[R R]=2.1 ; 95 \%$ CI: $1.2-3.5)$, diabetes mellitus $(R R=2.0$; 95\% CI: $1.2-3.3$ ), and high-grade stenosis ( $>60 \%$ or occlusion) in either artery, ipsilateral ( $\mathrm{RR}=1.9 ; 95 \% \mathrm{CI}: 1.2-3.0)$ or contralateral ( $\mathrm{RR}=1.7$; $95 \% \mathrm{CI}: 1.0-2.8)$.

Atherosclerotic RAS is associated with decreased kidney function, renal atrophy, and ultimately, renal failure. In a series of 204 kidneys with ARAS in 122 subjects followed by duplex scans for an average of 33 months, the 2-year cumulative incidence of renal atrophy was $5.5 \%$ in normal renal arteries, $11.7 \%$ with $<60 \%$ stenosis, and $20.8 \%$ with $60 \%$ stenosis $(P=0.009, \log$ rank test $) .{ }^{28}$ The authors also identified several baseline factors associated with a high risk of renal atrophy including hypertension, severe stenosis, and low renal cortical blood flow velocity. The occurrence of renal atrophy was well-correlated with increases in serum creatinine concentrations. This decline in organ function was also observed in a prospective study of patients with ARAS who were treated medically. ${ }^{29}$ Over the average follow-up period of 28 weeks, $46 \%$ had increased serum creatinine, $25 \%$ to $50 \%$ had a decline in GFR, and $37 \%$ had a decrease in kidney size by more than $10 \%$. It is not surprising then, that in a population of patients requiring dialysis, $12 \%$ likely developed end stage renal disease (ESRD) secondary to ARAS..$^{30}$ To make matters worse, ESRD due to ARAS is 
associated with worse survival rates as compared to other etiologies. The median survival rates in ESRD secondary to various etiologies was 25 months, 55 months, and 133 months for renovascular disease, malignant hypertension, and polycystic kidney disease, respectively. ${ }^{31}$ Increased mortality associated with ARAS has been observed even in the absence of ESRD with 2-year survival rates of $96 \%, 74 \%$, and $47 \%$ for unilateral RAS, BRAS, and RAS affecting a solitary functioning kidney, respectively. ${ }^{32}$ It is evident that severity of disease also predicts survival. Four-year adjusted survival for patients with $50 \%, 75 \%$, and $\geq 95 \%$ stenosis is $70 \%, 68 \%$, and a dismal 48\%, respectively. Furthermore, bilateral disease has been associated with a 4-year survival of $47 \%$ as compared with $59 \%$ for patients with unilateral disease $(P<0.001){ }^{19}$

\section{Diagnosis}

There is a wide array of diagnostic modalities available for the identification of atherosclerotic RAS. According to the American College of Cardiology/American Heart Association (ACC/AHA) Clinical Practice Guidelines, duplex ultrasonography, computed tomographic angiography (CTA), and magnetic resonance angiography (MRA) all receive a class I indication (level B evidence) as a screening test to establish the diagnosis of RAS. ${ }^{33}$ When the clinical index of suspicion is high and the results of noninvasive tests are inconclusive, catheter angiography is then recommended for screening as well. Each of these diagnostic techniques comes with its own unique set of advantages and disadvantages, providing clinicians with a number of approaches for any given patient. Receiving a Class III recommendation is captopril renography (also known as captopril scintigraphy), an older diagnostic modality widely used in the past. ${ }^{33}$ However, due to significant limitations and a sensitivity and specificity inferior to modern diagnostic techniques, this modality has fallen out of favor. Current guidelines state that captopril renal scintigraphy is not recommended as a screening test to diagnose RAS (level C evidence). ${ }^{33}$

Duplex ultrasonography combines direct visualization of renal arteries (B-mode imaging) with Doppler velocity measurements of blood flow. Information provided by this technique includes location and degree of stenosis, measurement of kidney size, visualization of adjacent processes (obstruction, masses, AAA, etc), and assessment of intrinsic small vessel renovascular disease. In addition, new software has allowed for visualization of the entire renal artery including the distal portion; however, ability to visualize accessory renal arteries remains limited. Duplex boasts a number of advantages. This noninvasive test is not affected by medications that the patient may be taking, the level of renal function, whether the disease is unilateral or bilateral, or if it affects a solitary functioning kidney. It is the least expensive imaging modality and does not require the use of intravenous contrast unlike CTA or MRA. Duplex is particularly useful for follow up after implantation of metallic stents. ${ }^{5}$ However, duplex is not without limitations. The test is time-consuming with prolonged examination times ( $\sim 5$ to 60 minutes). It can be technically challenging in obese patients or in the presence of abdominal gas, and there is also significant dependence on the operator. Notably, Hansen et al demonstrated a $10 \%$ to $20 \%$ rate of failure due to the operator's inexperience or the presence of obesity or bowel gas. ${ }^{34}$ To maximize results, an experienced technician should perform the test on a fasting patient in the morning. ${ }^{32,34}$ Detection of stenosis by ultrasound is determined by the measurement of a high peak systolic velocity ( $>180$ or $>200 \mathrm{~cm} /$ second) or by the renal aortic ratio, which is calculated by dividing the peak systolic velocity of the renal artery by the peak systolic velocity of the adjacent aorta. ${ }^{35} \mathrm{~A}$ normal ratio is $<3.5$. The sensitivity and specificity of a renal aortic ratio $>3.5$ as compared to contrast arteriography has been estimated at $84 \%$ and $97 \%$, respectively. ${ }^{35}$ This translates to a positive predictive value of $94 \%$ for the detection of stenosis greater than $60 \%$. A larger study by Olin et al, observed a higher value of $98 \%$ for both sensitivity and specificity. ${ }^{36}$

CTA is a form of diagnostic imaging that utilizes computer software to reconstruct an image from X-rays projected from several directions targeted at the same vessel of interest. Multidetector computed tomography (MDCT) boasts increased speed of image acquisition as well as higher spatial resolution. Initially, the image output is a series of overlapping axial images. Through post-processing of volumetric data, a three-dimensional angiographic representation is rendered. ${ }^{32}$ In addition to identifying stenotic lesions, CTA is useful for the visualization of adjacent anatomic structures. CTA has several advantages as compared to MRA, including higher spatial resolution and less artifact due to implanted metal stents; although vessel wall calcification can create potential difficulties in estimating the degree of stenosis. Compared to duplex, CTA is less operator-dependent. The requirement for injection of nephrotoxic iodinated contrast (100 to 150 cc) is a significant drawback, especially in the screening of ARAS, a population with increased prevalence of azotemia. While the time required for image acquisition is short, prolonged post-procedure processing time may be considered a limitation. CTA has proven to be a useful reliable test in the 
identification of RAS. For single-slice CTA, the sensitivity and the specificity for the detection of stenoses range between $88 \%-100 \%$ and $92 \%-98 \%$, respectively. ${ }^{37,38}$ For multidetector computed tomography, the sensitivity and specificity ranges from $86 \%-93 \%$ and $90 \%-100 \%$, respectively. ${ }^{39,40}$

MRA is a method of imaging that utilizes the property of nuclear magnetic resonance. The technique allows for the visualization of the target vessel structure without the need for ionizing radiation. Intravascular contrast is required to enhance the imaging of blood vessels; however, gadolinium contrast is less nephrotoxic than the ionized contrast used for CTA. Therefore, MRA can be performed in patients with some degree of renal insufficiency (though, not in patients with severe renal insufficiency or dialysis dependence due to the risk of nephrogenic systemic fibrosis with gadolinium contrast in those with a GFR $\leq 30 \mathrm{~mL} /$ minute $/ 1.73 \mathrm{~m}^{2}$ ), congestive heart failure, and dye allergy. Like CTA, MRA is less operator-dependent as compared to ultrasonography. MRA also contributes additional information not provided by other techniques. For example, MRA allows for the visualization of surrounding anatomical structures including accessory renal arteries that are otherwise not seen on ultrasonography. Furthermore, renal perfusion may be assessed, and GFR can be determined via calculation of gadolinium clearance. Unfortunately, MRA is the most expensive modality. In addition to cost, MRA has several other limitations. MRA cannot be used for patients with pacemakers or other metallic objects. Accordingly, MRA cannot be used to evaluate in-stent restenosis. While the aorta and proximal portion of the renal artery do not significantly move with respiration, persistent craniocaudal motion of the distal segment limits imaging in this portion of the renal artery. ${ }^{41}$ While ARAS is typically a disease of the proximal renal artery, stenosis due to FMD is often distally located and accuracy with MRA may be limited. ${ }^{32}$ The sensitivity and specificity for the identification of RAS have been shown to be greater than $90 \%$ by several studies. ${ }^{42-44}$ According to a meta-analysis of 25 studies published between 1985 to 2001 , the sensitivity and specificity of non-enhanced MRA were 94\% (95\% CI: 90\%-97\%) and 85\% (95\% CI: 82\%-87\%), respectively. ${ }^{45}$ For gadoliniumenhanced MRA, sensitivity was 97\% (95\% CI: 93\%-98\%) and specificity was 93\% (95\% CI: 91\%-95\%). Thus, specificity and positive predictive value were significantly better for gadolinium-enhanced MRA $(P<0.001)$.

While arterial angiography remains the gold standard for the diagnosis of RAS, it is typically used only after a positive noninvasive screening test. Angiography is also recommended in cases in which RAS is highly suspected and definitive noninvasive imaging cannot be obtained or in cases in which peripheral access is already obtained such as for imaging of the coronary arteries or abdominal aorta. ${ }^{33}$ Although more invasive than other techniques, the risk of complications is low. These risks include access-related complications, embolization, contrast-related allergic reactions, and contrast-induced nephropathy. The risk of contrast-induced nephropathy is $<3 \%$ in patients without diabetes or chronic kidney disease; though caution is warranted in patients with either of these conditions as the risk is increased dramatically. ${ }^{33}$

\section{Treatment}

Medical therapy is a central pillar in the approach to treatment of RAS. Major goals include glycemic control optimization, cholesterol reduction, smoking cessation, blood pressure reduction, and primary prevention with aspirin, if indicated. According to ACC/AHA guidelines, ACE inhibitors, angiotensin receptor blockers, calcium channel blockers, and beta-blockers all receive a class I indication for the treatment of hypertension associated with RAS. ${ }^{33}$ ACE inhibitors/angiotensin receptor blockers are a mainstay of most hypertensive regimens and have been shown to be $86 \%$ to $92 \%$ effective. ${ }^{1}$ A limitation of modulation of the renin-angiotensin system is the potential to induce acute renal failure in patients with RAS to solitary functioning kidneys, severe bilateral stenoses, or advanced chronic kidney disease. While management of hypertension is a primary goal in the treatment of RAS, care should be taken to avoid reducing perfusion pressure so low as to induce ischemic nephropathy in pressure-dependent kidneys. Another major drawback of medical therapy is the apparent inability to prevent progression of existing stenosis, which has been the primary impetus for the development of more permanent and decisive solutions such as surgical and percutaneous revascularization.

Revascularization of the renal artery can be accomplished surgically or endovascularly. Bypass grafts, aortorenal or nonanatomic, and the more technically challenging aortorenal endarterectomy make up the various surgical approaches to revascularization. These techniques are effective treatment options and are comparable to balloon angioplasty; however, major complications associated with surgery have been reported to be twice as common as compared to the endovascular approach. ${ }^{46}$ For this reason, angioplasty has largely replaced surgical therapy as a first-line treatment. To assess the efficacy of stent placement, angioplasty with stent placement was compared to angioplasty alone in a 
randomized prospective trial of patients with ostial ARAS. ${ }^{47}$ The results favored angioplasty with stent implantation in regards to rates of primary patency, restenosis, and need for intervention at 6 months. Currently, clinical guidelines endorse stent implantation in ostial lesions in patients with an established indication for revascularization (class I recommendation, level of evidence: B). ${ }^{33}$ An example of a severely stenotic renal artery before and after successful angioplasty and stenting is shown in Figure 1.

Endovascular interventions have been used, with limited success, to treat the three clinical syndromes associated with RAS: ischemic nephropathy, hypertension, and destabilizing cardiac syndromes. The treatment of asymptomatic RAS is not well established in an asymptomatic unilateral RAS in a viable kidney; though, it may be considered in a BRAS or unilateral RAS of a solitary viable kidney, a Class IIb indication (level of evidence: C). Specific instances of severe hypertension receive a Class IIa indication for revascularization. Guidelines state that percutaneous revascularization is reasonable for patients with RAS and accelerated hypertension, resistant hypertension, malignant hypertension, hypertension with an unexplained unilateral small kidney, and hypertension with intolerance to medication (level of evidence: B). Revascularization for the preservation of renal function also receives a Class IIa recommendation. Intervention can be considered for patients with RAS and progressive chronic kidney disease with BRAS or a RAS to a solitary functioning kidney (level of evidence: B). Intervention may also be considered for patients with chronic renal insufficiency with unilateral RAS, a Class IIb indication (level of evidence: C). RAS with UA is a class IIa indication. Currently, the only class I indication for catheter-based intervention is for patients with hemodynamically significant RAS and recurrent, unexplained congestive heart failure or flash pulmonary edema (level of evidence: B). Current guidelines endorse few indications for endovascular intervention in the treatment of clinical syndromes related to RAS due to a lack supporting clinical evidence. ${ }^{33}$

\section{Clinical trials}

Several major clinical trials have largely shaped our current understanding of the effectiveness of percutaneous intervention as compared to conservative treatment. Among the first large clinical trials was the DRASTIC trial. ${ }^{27}$ In this study, 106 patients with RAS ( $\geq 50 \%$ ) and resistant hypertension were randomized to renal angioplasty versus medical therapy. At 3 months, blood pressures were similar in the two groups, $169 \pm 28$ and $99 \pm 12 \mathrm{mmHg}$ in the angioplasty group and $176 \pm 31$ and $101 \pm 14 \mathrm{mmHg}$ in the drug therapy group $(P=0.25$ for systolic blood pressure, $P=0.36$ diastolic blood pressure). At 12 months, there were still no significant differences in blood pressures, diastolic or systolic. The authors concluded that in the treatment of patients with hypertension and RAS, angioplasty has little advantage over antihypertensive drug therapy. As is the theme with the existing body of clinical data, this trial has significant limitations. First, a sample size of 106 patients is less than ideal and possibly insufficient to see the true effect of invasive intervention. Notably, this trial examined the effectiveness of angioplasty alone, which has been shown to be inferior to angioplasty with stenting. ${ }^{46}$ Therefore, the therapy evaluated by this trial does not reflect current standard practice. Furthermore, RAS was defined as greater than 50\%, indicating that many non-hemodynamically significant lesions were treated. Finally, 22 out of the 55 patients originally randomized to medical therapy crossed over to the angioplasty arm because of persistent hypertension despite treatment with three or more drugs or because of deterioration of renal function. These patients were still analyzed as intention-to-treat (ITT). While the lack of benefit observed in this trial may be real, multiple flaws inherent in the study design prevent any convincing conclusions from being drawn.

Another major clinical trial is known as the STent placement and blood pressure and lipid-lowering for the prevention of progression of renal dysfunction caused by Atherosclerotic ostial stenosis of the Renal artery (STAR) trial. ${ }^{48}$ STAR was a multicenter randomized study of 140 patients with RAS. Inclusion criteria included renal impairment (GFR $<80$ $\mathrm{mL} / \mathrm{minute})$ and well-controlled blood pressure $(<140 / 90$ $\mathrm{mmHg}$ ) on a stable medication regimen for 1 month prior to enrollment. Patients were randomized to renal artery stenting and medical therapy or medical therapy alone. The primary outcome was a $>20 \%$ decrease in creatinine clearance during 2 years of follow-up. Ten of 64 patients (16\%) in the stent placement group and 16 patients $(22 \%)$ in the medication group reached the primary endpoint (hazard ratio: 0.73 ; 95\% CI: 0.33-1.61). Several serious complications occurred in the stent group including two procedurerelated deaths $(3 \%)$, one late death secondary to an infected hematoma, and one patient required dialysis secondary to cholesterol embolism. There was no difference between the two groups in regards to other secondary endpoints: changes in blood pressure, incidence of refractory or malignant hypertension and pulmonary edema, cardiovascular morbidity and mortality, and total mortality. The authors concluded stent placement with medical treatment had no clear effect on progression of impaired renal function but led to a small number of significant procedure-related complications. They 
state that their findings favor a conservative approach to patients with ARAS, focused on cardiovascular risk factor management. Unfortunately, STAR represents yet another trial plagued with bias and design flaws. Of the 140 patients, $33 \%$ had only mild RAS (50\%-70\%). Furthermore, 12 of 64 patients (19\%) in the stenting arm had RAS $<50 \%$ and did not receive a stent but were still analyzed in ITT. An additional six patients in the stent arm did not receive a stent (one received balloon angioplasty, one died before placement, two declined the stent, and two technical failures) but all were analyzed in ITT. It is also worth noting that $>50 \%$ had unilateral disease. This is important because bilateral disease is usually required to observe changes in creatinine clearance, which was the primary endpoint.

The third and largest clinical trial to date is known as Angioplasty and Stenting for Renal Artery Lesions (ASTRAL), a multicenter, prospective randomized study of 806 patients with uncontrolled or refractory hypertension or unexplained renal dysfunction with concomitant imaging to suggest RAS. ${ }^{49}$ The primary outcome was change in renal function measured by mean slope of the reciprocal of a serum creatinine curve. Secondary outcomes were change in blood pressure, time to renal and major cardiovascular events, and mortality. Over a 5-year period, the rate of progression of renal impairment was $-0.07 \times 10^{-3} \mathrm{~L} / \mu \mathrm{mol}$ per year in the revascularization group and $-0.13 \times 10^{-3} \mathrm{~L} / \mu \mathrm{mol}$ per year in the medical therapy group. This was a difference favoring revascularization of $0.06 \times 10^{-3} \mathrm{~L} / \mu \mathrm{mol}$ per year $(95 \%$ CI: -0.002 to $0.13 ; P=0.06)$. Over the same time period, the mean serum creatinine level was $1.6 \mu \mathrm{mol} / \mathrm{L}$ lower in the revascularization group than in the medical therapy group (95\% CI: -8.4 to 5.2 [0.02 mg per deciliter; 95\% CI: -0.10 to 0.06$]$ ). In regards to secondary endpoints, there was no significant difference in systolic blood pressure, while the decrease in diastolic blood pressure was smaller in the revascularization group. Similar rates of renal and major cardiovascular events were seen in both groups with hazard ratios of 0.97 (95\% CI: $0.67-1.40 ; P=0.88)$, and $0.94(95 \%$ CI: $0.75-1.19 ; P=0.61)$, respectively. Several serious complications associated with revascularization were observed in 23 patients including two deaths, and three amputations of toes or limb. From these results, the author's concluded that there was no evidence of a worthwhile clinical benefit from revascularization in patients with atherosclerotic renovascular disease. Selection bias is of particular concern in this trial. For patients to be enrolled, the treating physician had to determine that the patient was reasonable to be randomized, meaning it was uncertain if the subject would benefit from revascularization. If an individual was thought to need revascularization within 6 months, they were excluded. Ultimately, only $83 \%$ randomized to stenting actually underwent the procedure. Interestingly, one-quarter of patients had normal renal function at baseline and $41 \%$ had $<70 \%$ stenosis, but renal function was the primary outcome. It is concerning that recruitment took 7 years, and $42 \%$ of all sites only randomized between one to five patients while $61 \%$ randomized nine or fewer patients. Furthermore, the major adverse event rate at 24 hours was $9 \%$, while the usual accepted rate is $2 \%$.

The Cardiovascular Outcomes in Renal Atherosclerotic Lesions (CORAL) trial is a multicenter, randomized trial sponsored by the National Heart, Lung, and Blood Institute with results published in early 2014 in the New England Journal of Medicine. ${ }^{50}$ CORAL was designed to compare optimum medical therapy alone to stenting with optimum medical therapy and is thought to have corrected for flaws inherent in previous trials of its kind. The primary outcome is a composite endpoint of major cardiovascular and renal adverse events. To be included in the trial, patients were required to have atherosclerotic renal stenosis of $\geq 60 \%$ with a systolic pressure gradient of $20 \mathrm{mmHg}$ or stenosis $\geq 80 \%$ with no gradient necessary and systolic hypertension of $\geq 155 \mathrm{mmHg}$ on at least two antihypertensive medications. After a median follow-up period of 43 months, the authors found no significant difference between the two groups in regards to the composite endpoint, any of the individual components of the composite endpoints, or all-cause mortality.

In summary, the major trials published to date have been unable to prove a clinical benefit of endovascular revascularization for the treatment of RAS. However, significant design flaws and selection bias plague many of these studies, thus, limiting the usefulness of available data. While a truly definitive answer remains elusive, the most recent data from CORAL is more convincing than that of previous studies, thus favoring medical therapy alone over stenting for the treatment of ARAS. Ongoing and future studies are likely to provide additional constructive data, further informing clinical decision-making.

\section{Need for clinical predictors}

Despite a lack supporting data, marginal benefits seen in some studies may be more pronounced if appropriate subgroups were to be identified. Presumably, such patients would consistently benefit from revascularization. Several clinical predictors of successful outcomes following revascularization have been proposed. 
Rocha-Singh et al followed 150 consecutive hypertensive patients with 180 renal artery lesions ( $\geq 75 \%$ stenosis) after undergoing renal artery stenting. ${ }^{51}$ Multivariate logistic regression analysis was used to identify clinical variables related to improved blood pressure control at a mean follow-up of 13 months. Only BRAS or mean arterial pressure $>110$ predicted blood pressure improvement. While brain natriuretic peptide (BNP) is already a well-known marker of congestive heart failure and major cardiovascular events, it may also play a role in predicting outcomes following RAS. Though initially released from the myocardium in response to myocyte stretch, BNP's primary physiological site of action is in the kidney. Interestingly, angiotensin II has been implicated in inducing the production of BNP. ${ }^{52}$ In a study of 27 patients with refractory hypertension and significant RAS, BNP was measured before and after renal artery stent placement. ${ }^{53}$ The authors observed an elevated BNP level in patients with significant RAS, and results indicated that a baseline BNP $>80 \mathrm{pg} / \mathrm{mL}$ appears to predict blood pressure response after successful stent revascularization.
Elevated pulse pressure (PP) has been used as a predictor of increased cardiovascular events, and evidence suggests that PP may help to predict outcomes following RAS revascularization as well. A retrospective study at the Loyola University Medical Center found that a low PP was associated with improvements and stabilizations in renal function as well as improvements and stabilizations in blood pressure. ${ }^{54}$ The authors concluded that a wide PP may reflect more advanced vascular stiffness and renal disease, distinguishing patients less likely to benefit from revascularization.

Poor treatment response following revascularization might be explained by irreversible microvascular disease downstream of stenotic lesions secondary to longstanding hypertension. Hypertension induced glomerulosclerosis and nephrosclerosis may increase the resistance to flow through both affected and unaffected kidneys. An increase in resistance could, therefore, serve as a marker of structural alterations of the renal microvasculature. Clinically, increased intrarenal resistance can be observed by less diastolic flow and a more resistant waveform. The resistive index quantifies this

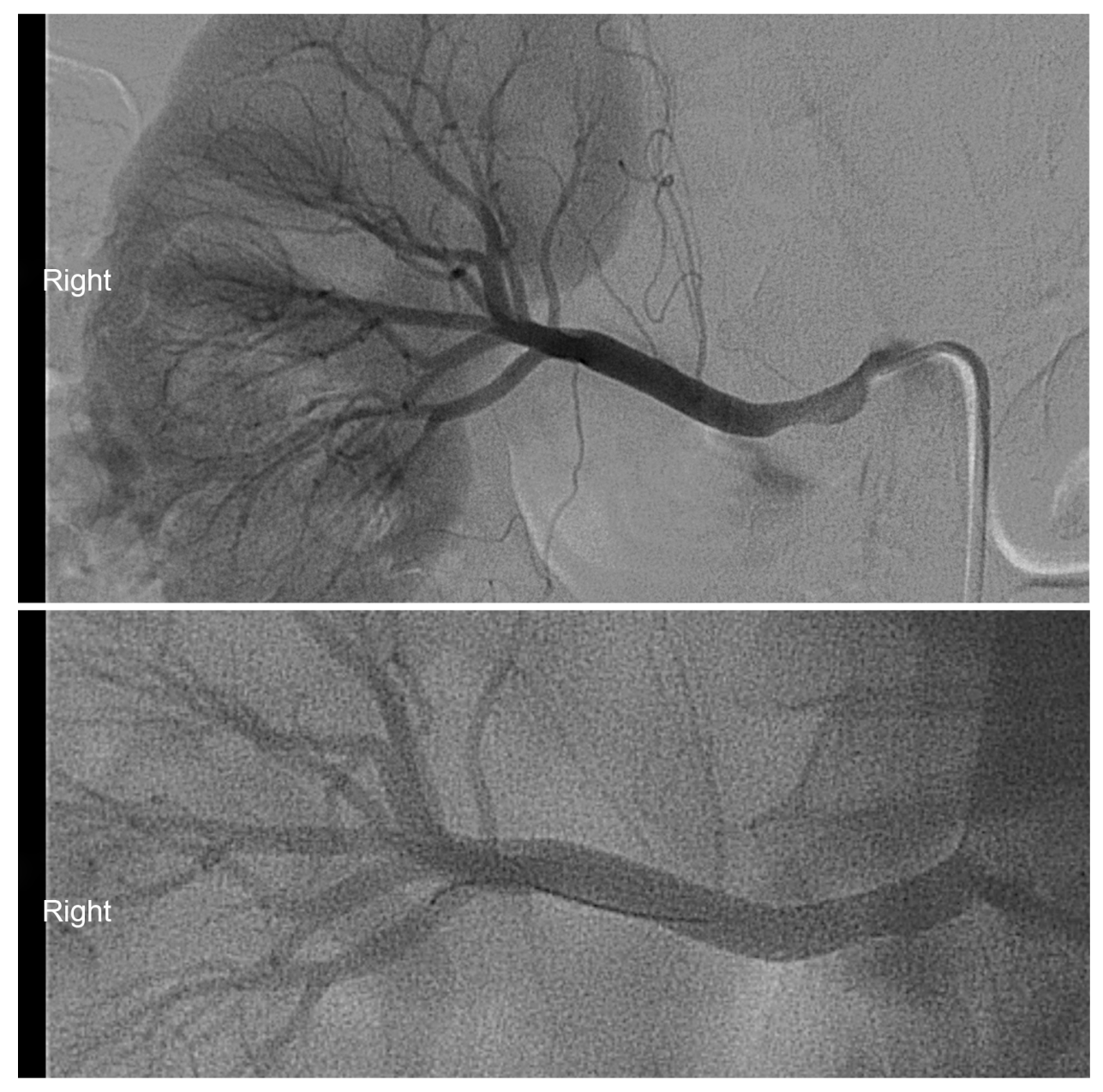

Figure I Severe renal artery stenosis before (top) and after (bottom) angioplasty and stenting. 
observation with the formula $[1-$ (end-diastolic velocity $\div$ maximal systolic velocity)] $\times 100$. In 2001, Radermacher et al tested the hypothesis in 131 patients who underwent renal artery stent implantation. ${ }^{55}$ It was concluded that a resistive index value $\geq 80$ reliably identifies patients in whom invasive intervention will not improve renal function, blood pressure, or kidney survival. A study published 2 years later observed results to the contrary. ${ }^{56}$ One hundred seventy-six patients with severe $(\geq 70 \%)$ ostial ARAS were divided into three strata of resistive indices, $<0.7,0.7-0.8$, and $>0.8$. All groups underwent renal artery stenting and all groups had similar reductions in blood pressure at discharge and similar improvements in renal function at 6-month follow-up.

The measurement of fractional flow reserve (FFR) is a technique commonly used in the evaluation of coronary artery lesions. FFR measures pressure differences across specific arterial lesions obtained during maximum hyperemia and is a marker of the severity of vascular disease. More recently, the technique was adapted to study renal arteries. ${ }^{57}$ Using papaverine (endothelium independent vasodilator) to induce hyperemia of the renal vasculature of 13 patients, investigators found no correlation between blood pressure gradient, hyperemic pressure gradient, or FFR with angiographic stenosis. The best correlation was found between hyperemic pressure gradient and FFR $(r=0.94)$. The poor correlation between angiographic stenosis and hemodynamic parameters implies that angiography alone provides insufficient diagnostic information.

Renal frame count (RFC) is a method of assessing and quantifying perfusion of the kidneys in the setting of RAS. By utilizing the frame counter on standard angiographic images, one can count the number of frames required for dye to pass through the renal parenchyma and reach a distally defined point. It has been hypothesized that following revascularization, improvement in two variables of renal perfusion, RFC and renal blush grade, would correlate with improvement in renovascular hypertension. ${ }^{58}$ This hypothesis was tested in a series of 24 patients with unilateral RAS who underwent revascularization. The authors found that the RFC and renal blush grade were in fact impaired in RAS and improved following renal artery stenting. Specifically, an improvement in RFC $>4$ was associated with lower blood pressures. Though not statistically significant, they also found that a baseline RFC $\geq 25$ was $75 \%$ predictive of response to renal stent therapy. An optimal baseline RFC predictive of clinical response may be better defined by a larger study.

Characterization of renal artery plaques through intravascular ultrasound with virtual histology (VH-IUVS) has also shown promise in identifying a population potentially responsive to intervention. In a series of 25 patients who underwent VH-IUVS prior to renal artery revascularization, investigators found that a larger necrotic core was significantly associated with deterioration in estimated GFR following intervention. ${ }^{59}$ Percentage of necrotic core has also been linked to a lack of improvement in RFC following RAS stenting. A similar series utilized VH-IUVS to characterize the stenotic arterial segment prior to revascularization, while RFC was measured before and after intervention. ${ }^{60}$ The results demonstrated an association between increased necrotic core and lack of improvement in RFC following revascularization.

\section{Cardiac destabilizing syndromes}

While improvements in hypertension and renal function following revascularization of RAS remain elusive, it is important to remember the clear benefits seen in the treatment of cardiac destabilizing syndromes. As previously mentioned, flash pulmonary edema in the setting of hemodynamically significant RAS has earned the only Class I indication for revascularization according to current guidelines (ACC/ AHA). ${ }^{33}$ In the case of bilateral stenosis, the goal of stent placement is the restoration of renal blood flow, thus promoting the return of natriuresis. In addition, patients whose kidney function is no longer renin-dependent may be started on ACE inhibitors, thus improving long-term survival in the context of heart failure. In unilateral stenosis, reperfusion and reversal of renal ischemia removes the stimulus for angiotensin release, preventing acute increases in left ventricular afterload. This logic appears to be supported by convincing clinical data demonstrating benefits of revascularization. Koshla et al analyzed prospective registry data of patients with refractory hypertension and hemodynamically significant RAS presenting with UA (20 patients) or congestive heart failure (28 patients). ${ }^{61}$ All patients underwent renal artery stent implantation with or without coronary intervention. Results indicated a benefit for revascularization, independent of having received PCI. New York Heart Association classification was improved in congestive heart failure patients and Canadian Cardiovascular Society angina classification was improved in patients with UA. Both effects were sustained in $73 \%$ of patients at a mean follow-up of 8.4 months. Bloch et al ${ }^{9}$ reported similar results from a retrospective analysis of 90 patients ( $41 \%$ BRAS, $12 \%$ unilateral RAS) with pulmonary edema. Following revascularization, more than three-quarters of patients with BRAS and one-third of patients with unilateral RAS remained free of pulmonary 
edema. In addition, investigators have demonstrated reduction in hospitalizations due to heart failure, improvement in New York Heart Association functional classification, and increased utilization of ACE inhibitors following RAS revascularization. ${ }^{62}$

\section{Conclusion}

RAS is a prevalent disease commonly encountered in association with other atherosclerotic vascular disease. RAS is associated with poor prognosis in terms of lesion progression, decline of renal function, and overall mortality. The detection of RAS can be accomplished via several effective diagnostic modalities, the choice of which can be tailored to the needs of individual patients. Once identified, the next step in management of RAS is unclear. Renal artery stenting is a therapy that has shown great promise, but has, thus far, not lived up to its expected and much hoped for potential. More outcome studies are needed to justify routine renal artery stenting for hypertension or chronic kidney disease. Better clinical predictors are needed to help with patient selection in hopes of identifying subgroups that might more consistently benefit from renal artery revascularization. Finally, it is important to recognize the well-established benefits of percutaneous intervention seen in the treatment of cardiac destabilizing syndromes.

\section{Disclosure}

The authors report no conflicts of interest in this work.

\section{References}

1. Safian RD, Textor SC. Renal-artery stenosis. N Engl J Med. 2001;344(6): 431-442.

2. Blum U, Krumme B, Flügel P, et al. Treatment of ostial renal-artery stenoses with vascular endoprostheses after unsuccessful balloon angioplasty. N Engl J Med. 1997;336:459-465.

3. Burket MW, Cooper CJ, Kennedy DJ, et al. Renal artery angioplasty and stent placement: predictors of a favorable outcome. Am Heart J. 2000;139:64-71.

4. Slovut DP, Olin JW. Fibromuscular dysplasia. $N$ Engl J Med. 2004;350(18):1862-1871.

5. Dieter R, Schmidt W, Pacanowski J, Jaff MR. Renovascular hypertension. Expert Rev Cardiovasc Ther. 2005;3(3):413-422.

6. Gottam N, Nanjundappa A, Dieter R. Renal artery stenosis: pathophysiology and treatment. Expert Rev Cardiovasc Ther. 2009;7(11): 1413-1420.

7. Goldblatt H, Lynch J, Hanzel RF, Summerville WW. Studies on experimental hypertension I: the production of persistent elevation of systolic blood pressure by means of renal ischemia. J Exp Med. 1934;59(3):347-379.

8. Textor SC. Ischemic nephropathy: where are we now? J Am Soc Nephrol. 2004;15(8):1974-1982.

9. Bloch MJ, Trost DW, Pickering TG, Sos TA, August P. Prevention of recurrent pulmonary edema in patients with bilateral renovascular disease through renal artery stent placement. Am J Hypertens. 1999;12 (1 Pt 1):1-7.
10. Pickering TG, Herman L, Devereux RB, et al. Recurrent pulmonary oedema in hypertension due to bilateral renal artery stenosis: treatment by angioplasty or surgical revascularisation. Lancet. 1988;2(8610):551-552.

11. Crowley J, Santos R, Peter R, et al. Progression of renal artery stenosis in patients undergoing cardiac catheterization. Am Heart J. 1998;136(5):913-918.

12. Hansen KJ, Edwards MS, Craven TE, et al. Prevalence of renovascular disease in the elderly: a population-based study. JVasc Surg. 2002;36(3): 443-451.

13. Conlon PJ, O'Riordan E, Kalra PA. New insights into the epidemiologic and clinical manifestations of atherosclerotic renovascular disease. $\mathrm{Am}$ J Kidney Dis. 2000;35(4):573-587.

14. Singh M, Morshedi-Meibodi A, Steen L, Dieter RS. Overlap of atherosclerotic disease. In: Dieter RS, Dieter R III, Dieter R Jr, editors. Peripheral Arterial Disease. New York: McGraw-Hill Professional; 2009:177-184

15. Wollenweber J, Sheps S, Davis G. Clinical course of atherosclerotic renovascular disease. Am J Cardiol. 1968;21(1):60-71.

16. Weber-Mzell D, Kotanko P, Schumacher M, Klein W, Skrabala F. Coronary anatomy predicts presence or absence of renal artery stenosis: a prospective study in patients undergoing cardiac catheterization for suspected coronary artery disease. Eur Heart J. 2002;23(21): 1684-1691.

17. Rihal CS, Textor SC, Breen JF, et al. Incidental renal artery stenosis among a prospective cohort of hypertensive patients undergoing coronary angiography. Mayo Clin Proc. 2002;77(4):309-316.

18. Valentine R, Clagett G, Miller G, Myers S, Martin J, Chervu A. The coronary risk of unsuspected renal artery stenosis. J Vasc Surg. 1993;18(3):433-439; discussion 439-440.

19. Conlon PJ, Little MA, Pieper K, Mark DB. Severity of renal vascular disease predicts mortality in patients undergoing coronary angiography. Kidney Int. 2001;60(4):1490-1497.

20. Olin JW, Melia M, Young JR, Graor RA, Risius B. Prevalence of atherosclerotic renal artery stenosis in patients with atherosclerosis elsewhere. Am J Med. 1990;88(1N):46N-51N.

21. Louie J, Isaacson J, Zierler R, Bergelin R, Strandness D. Prevalence of carotid and lower extremity arterial disease in patients with renal artery stenosis. Am J Hypertens. 1994;7(5):436-439.

22. Zierler R, Bergelin R, Polissar N, et al. Carotid and lower extremity arterial disease in patients with renal artery atherosclerosis. Arch Intern Med. 1998;158(7):761-767.

23. Rossi G, Rossi A, Zanin L, et al. Excess prevalence of extracranial carotid artery lesions in renovascular hypertension. Am J Hypertens. 1992;5(1):8-15.

24. Kuroda S, Nishida N, Uzu T, et al. Prevalence of renal artery stenosis in autopsy patients with stroke. Stroke. 2000;31(1):61-65.

25. Caps MT, Perissinotto C, Zierler RE, et al. Prospective study of atherosclerotic disease progression in the renal artery. Circulation. 1998;98(25):2866-2872.

26. Schreiber MJ, Pohl MA, Novick AC. The natural history of atherosclerotic and fibrous renal artery disease. Urol Clin North Am. 1984;11(3):383-392.

27. van Jaarsveld BC, Krijnen P, Pieterman H, et al. The effect of balloon angioplasty on hypertension in atherosclerotic renal-artery stenosis. Dutch Renal Artery Stenosis Intervention Cooperative Study Group. N Engl J Med. 2000;342(14):1007-1014.

28. Caps MT, Zierler RE, Polissar NL, et al. Risk of atrophy in kidneys with atherosclerotic renal artery stenosis. Kidney Int. 1998;53(3):735-742.

29. Dean RH, Kieffer RW, Smith BM, et al. Renovascular hypertension: anatomic and renal function changes during drug therapy. Arch Surg. 1981;116(11):1408-1415.

30. Mailloux LU, Napolitano B, Bellucci AG, Vernace M, Wilkes BM, Mossey RT. Renal vascular disease causing end-stage renal disease, incidence, clinical correlates, and outcomes: a 20-year clinical experience. Am J Kidney Dis. 1994;24(4):622-629.

31. Mailloux LU, Bellucci AG, Mossey RT, et al. Predictors of survival in patients undergoing dialysis. Am J Med. 1988;84(5):855-862. 
32. Guttormsen B, Gimelli G. Renal artery disease. In: Dieter RS, Dieter R III, Dieter R Jr, editors. Peripheral Arterial Disease. New York: McGraw-Hill Professional; 2009:619-638.

33. Hirsch AT, Haskal ZJ, Hertzer NR, et al. American Association for Vascular Surgery; Society for Vascular Surgery; Society for Cardiovascular Angiography and Interventions; Society for Vascular Medicine and Biology; Society of Interventional Radiology; ACC/AHA Task Force on Practice Guidelines Writing Committee to Develop Guidelines for the Management of Patients With Peripheral Arterial Disease; American Association of Cardiovascular and Pulmonary Rehabilitation; National Heart, Lung, and Blood Institute; Society for Vascular Nursing; TransAtlantic Inter-Society Consensus; Vascular Disease Foundation. ACC/AHA 2005 Practice Guidelines for the management of patients with peripheral arterial disease (lower extremity, renal, mesenteric, and abdominal aortic): a collaborative report from the American Association for Vascular Surgery/Society for Vascular Surgery, Society for Cardiovascular Angiography and Interventions, Society for Vascular Medicine and Biology, Society of Interventional Radiology, and the ACC/AHA Task Force on Practice Guidelines (Writing Committee to Develop Guidelines for the Management of Patients With Peripheral Arterial Disease): endorsed by the American Association of Cardiovascular and Pulmonary Rehabilitation; National Heart, Lung, and Blood Institute; Society for Vascular Nursing; TransAtlantic Inter-Society Consensus; and Vascular Disease Foundation. Circulation. 2006;113(11):e463-e654.

34. Hansen KJ, Tribble RW, Reavis SW, et al. Renal duplex sonography: evaluation of clinical utility. J Vasc Surg. 1990;12(3):227-236.

35. Taylor DC, Kettler MD, Moneta GL, et al. Duplex ultrasound scanning in the diagnosis of renal artery stenosis: a prospective evaluation. J Vasc Surg. 1988;7(2):363-369.

36. Olin JW, Piedmonte MR, Young JR, DeAnna S, Grubb M, Childs MB. The utility of duplex ultrasound scanning of the renal arteries for diagnosing significant renal artery stenosis. Ann Intern Med. 1995;122(11):833-838.

37. Wittenberg G, Kenn W, Tschammler A, Sandstede J, Hahn D. Spiral CT angiography of renal arteries: comparison with angiography. Eur Radiol. 1999;9(3):546-551.

38. Galanski M, Prokop M, Chavan A, Schaefer C, Jandeleit K, Olbricht C. [Accuracy of CT angiography in the diagnosis of renal artery stenosis]. Rofo. 1994;161(6):519-525. German.

39. Hahn U, König CW, Miller S, et al. [Multidetector CT Angiography - is it a valuable screening tool to detect significant renal artery stenosis?]. Rofo. 2001;173(12):1086-1092. German.

40. Willmann JK, Wildermuth S, Pfammatter T, et al. Aortoiliac and renal arteries: prospective intraindividual comparison of contrast-enhanced three-dimensional MR angiography and multi-detector row CT angiography. Radiology. 2003;226(3):798-811.

41. Vasbinder GB, Maki JH, Nijenhuis RJ, et al. Motion of the distal renal artery during three-dimensional contrast-enhanced breath-hold MRA. J Magn Reson Imaging. 2002;16(6):685-696.

42. Snidow JJ, Johnson MS, Harris VJ, et al. Three-dimensional gadolinium-enhanced MR angiography for aortoiliac inflow assessment plus renal artery screening in a single breath hold. Radiology. 1996;198(3):725-732.

43. Rieumont MJ, Kaufman JA, Geller SC, et al. Evaluation of renal artery stenosis with dynamic gadolinium-enhanced MR angiography. AJR Am J Roentgenol. 1997;169(1):39-44.

44. Fain SB, King BF, Breen JF, Kruger DG, Riederer SJ. High- spatialresolution contrast-enhanced MR angiography of the renal arteries: a prospective comparison with digital subtraction angiography. Radiology. 2001;218(2):481-490.
45. Tan KT, van Beek EJ, Brown PWG, van Delden OM, Tijssen J, Ramsay LE. Magnetic resonance angiography for the diagnosis of renal artery stenosis: a meta-analysis. Clin Radiol. 2002;57(7):617-624.

46. Weibull H, Bergqvist D, Bergentz SE, Jonsson K, Hulthén L, Manhem P. Percutaneous transluminal renal angioplasty versus surgical reconstruction of atherosclerotic renal artery stenosis: a prospective randomized study. J Vasc Surg. 1993;18(5):841-850; discussion 850-852.

47. Van de ven PJG, Kaatee R, Beurtler JJ, et al. Arterial stenting and balloon angioplasty in ostial atherosclerotic renovascular disease: a randomised trial. Lancet. 1999;353(9149):282-286.

48. Bax L, Woittiez AJ, Kouwenberg HJ, et al. Stent placement in patients with atherosclerotic renal artery stenosis and impaired renal function: a randomized trial. Ann Intern Med. 2009;150(12):840-841, W150.

49. Wheatley K, Ives N, Gray R, et al; ASTRAL Investigators. Revascularization versus medical therapy for renal-artery stenosis. N Engl J Med. 2009;361(20):1953-1962.

50. Cooper CJ, Murphy TP, Cutlip DE, et al; CORAL Investigators. Stenting and medical therapy for atherosclerotic renal-artery stenosis. $N$ Engl J Med. 2014;370(1):13-22.

51. Rocha-Singh KJ, Mishkel GJ, Katholi RE, et al. Clinical predictors of improved long-term blood pressure control after successful stenting of hypertensive patients with obstructive renal artery atherosclerosis. Catheter Cardiovasc Interv. 1999;47(2):167-172.

52. Wiese S, Breyer T, Dragu A, et al. Gene expression of brain natriuretic peptide in isolated atrial and ventricular myocardium: influence of angiotensin II and diastolic fiber length. Circulation. 2000;102(25): 3074-3079.

53. Silva JA, Chan AW, White CJ, et al. Elevated brain natriuretic peptide predicts blood pressure response after stent revascularization in patients with renal artery stenosis. Circulation. 2005;111(3):328-333.

54. Dieter RS, Darki A, Nanjundappa A, et al. Usefulness of Wide pulse pressure as a predictor of poor outcome after renal artery angioplasty and stenting. Am J Cardiol. 2009;104(5):732-734.

55. Radermacher J, Chavan A, Bleck J, et al. Use of Doppler ultra-sonography to predict the outcome of therapy for renal-artery stenosis. $N$ Engl $J$ Med. 2001;344(6):410-417.

56. Zeller T, Müller C, Frank U, et al. Stent angioplasty of severe atherosclerotic ostial renal artery stenosis in patients with diabetes mellitus and nephrosclerosis. Catheter Cardiovasc Interv. 2003;58(4): 510-515.

57. Subramanian R, White CJ, Rosenfield K, et al. Renal fractional flow reserve: a hemodynamic evaluation of moderate renal artery stenoses. Catheter Cardiovasc Interv. 2005;64(4):480-486.

58. Mitchell JA, Subramanian R, White CJ, et al. Predicting blood pressure improvement in hypertensive patients after renal artery stent placement: renal fractional flow reserve. Catheter Cardiovasc Interv. 2007;69(5):685-689.

59. Takumi T, Mathew V, Barsness, GW, et al. The association between renal atherosclerotic plaque characteristics and renal function before and after renal artery intervention. Mayo Clin Proc. 2011;86(12): 1165-1172.

60. Prasad A, Ilapakurti M, Hu P, et al. Renal artery plaque composition is associated with changes in renal frame count following renal artery stenting. J Invasive Cardiol. 2011;23(6):227-231.

61. Khosla S, White CJ, Collins TJ, Jenkins JS, Shaw D, Ramee SR. Effects of Renal Artery Stent Implantation in Patients with Renovascular Hypertension Presenting with Unstable Angina or Congestive Heart Failure. Am J Cardiol. 1997;80(3):363-366.

62. Gray BH, Olin JW, Childs MB, Sullivan TM, Bacharach JM. Clinical benefit of renal artery angioplasty with stenting for the control of recurrent and refractory congestive heart failure. Vasc Med. 2002;7(4): 275-279. 
International Journal of Nephrology and Renovascular Disease

Dovepress

\section{Publish your work in this journal}

The International Journal of Nephrology and Renovascular Disease is an international, peer-reviewed open-access journal focusing on the pathophysiology of the kidney and vascular supply. Epidemiology, screening, diagnosis, and treatment interventions are covered as well as basic science, biochemical and immunological studies. The journal welcomes

original research, clinical studies, reviews \& evaluations, expert opinion and commentary, case reports and extended reports. The manuscript management system is completely online and includes a very quick and fair peerreview system, which is all easy to use. Visit http://www.dovepress.com/ testimonials.php to read real quotes from published authors.

Submit your manuscript here: http://www.dovepress.com/international-journal-of-nephrology-and-renovascular-disease-journal 